\title{
MicroRNA miR-212-3p targets NFIA to suppress HBV replication and tumor progression in hepatocellular carcinoma via repressing Enhancer I activity
}

\section{Type}

Research paper

\section{Keywords}

HBV, hepatocellular carcinoma, miR-212-3p, NFIA

\begin{abstract}
Introduction

Emerging evidence identifies that microRNAs (miRNAs) are associated with hepatitis B virus (HBV) infection. In the current study, we mainly focus on the functions and underlying mechanisms of miR-212-3p in HBV replication in hepatocellular carcinoma (HCC).

\section{Material and methods}

The levels of miR-212-3p, nuclear factor I A (NFIA) and HBV DNA copies were measured by qRTPCR. The level of core particle-associated HBV DNA, the productions of hepatitis B surface antigen ( $\mathrm{HBsAg})$ and hepatitis B e-antigen ( $\mathrm{HBeAg})$, and the expression of NFIA were detected via southern blot assay, ELISA and western blot assay, respectively. The putative target of miR-212-3p was predicted by TargetScan and Pictar, followed by the dual luciferase reporter assay and RNA immunoprecipitation (RIP) assay to validate the interaction. The interaction between miR-212-3p and Enhancer I/X promoter (Enl/Xp) reporter was also verified by dual luciferase reporter assay. In addition, the cell viability and apoptotic rate were detected by MTT and flow cytometry, respectively.
\end{abstract}

\section{Results}

miR-212-3p mimics or NFIA knockdown inhibited HBV expression and replication in HepG2.2.15 cells, while miR-212-3p inhibitor or NFIA overexpression showed the opposite trend. NFIA was confirmed as a direct target of miR-212-3p. Furthermore, miR-212-3p impeded HBV expression and replication by suppressing NFIA. Besides, miR-212-3p lowered EnI/Xp activity by regulating NFIA. In addition, miR-212-3p retarded cell viability and induced apoptosis through targeting NFIA.

Conclusions

miR-212-3p targets NFIA to down-regulate its expression, thereby inhibiting HBV replication and tumorigenesis in HCC. Our finding might provide a promising therapeutic target for HBV infection. 
miR-212-3p targets NFIA to suppress HBV replication and tumor progression in hepatocellular carcinoma via repressing Enhancer I activity

\section{Hui Tian, Zhenkun He*}

Department of Infectious Disease, Huaihe Hospital of Henan University, Kaifeng 475000, China.

*Correspondence: Department of Infectious Disease, Huaihe Hospital of Henan University, No.115, Ximen Street, Gulou District, Kaifeng 475000, China.

Tel: +86-0371-23906643. E-mail: hezhenshenccn@163.com.

\section{Running title:}

miR-212-3p/NFIA axis regulates HBV replication. 


\section{Abstract}

Introduction: Emerging evidence identifies that microRNAs (miRNAs) are associated with hepatitis B virus (HBV) infection. In the current study, we mainly focus on the functions and underlying mechanisms of miR-212-3p in HBV replication in hepatocellular carcinoma (HCC).

Methods: The levels of miR-212-3p, nuclear factor I A (NFIA) and HBV DNA copies were measured by qRT-PCR. The level of core particle-associated HBV DNA, the productions of hepatitis B surface antigen (HBsAg) and hepatitis B e-antigen (HBeAg), and the expression of NFIA were detected via southern blot assay, ELISA and western blot assay, respectively. The putative target of miR-212-3p was predicted by TargetScan and Pictar, followed by the dual luciferase reporter assay and RNA immunoprecipitation (RIP) assay to validate the interaction. The interaction between miR-212-3p and Enhancer I/X promoter $(\mathrm{EnI} / \mathrm{Xp})$ reporter was also verified by dual luciferase reporter assay. In addition, the cell viability and apoptotic rate were detected by MTT and flow cytometry, respectively.

Results: miR-212-3p mimics or NFIA knockdown inhibited HBV expression and replication in HepG2.2.15 cells, while miR-212-3p inhibitor or NFIA overexpression showed the opposite trend. NFIA was confirmed as a direct target of miR-212-3p. Furthermore, miR-212-3p impeded HBV expression and replication by suppressing NFIA. Besides, miR-212-3p lowered EnI/Xp activity by regulating NFIA. In addition, miR-212-3p retarded cell viability and induced apoptosis through targeting NFIA.

Conclusion: miR-212-3p targets NFIA to down-regulate its expression, thereby 
inhibiting HBV replication and tumorigenesis in HCC. Our finding might provide a promising therapeutic target for HBV infection.

Keywords: miR-212-3p, NFIA, HBV, hepatocellular carcinoma 


\section{Introduction}

Hepatitis B virus (HBV), a major threat for liver cancer, has infected 350 million individuals worldwide and one-third resides in China $[1,2]$. HBV, a virus with partially double stranded DNA genome, is transcribed into four viral transcripts (3.5 $\mathrm{kb}, 2.4 \mathrm{~kb}, 2.1 \mathrm{~kb}$, and $0.7 \mathrm{~kb}$ ) [3]. Due to the small genome of HBV, the four HBV promoter/enhancers (preS1 promoter $(\mathrm{S} 1 \mathrm{p})$, preS1 promoter $(\mathrm{S} 2 \mathrm{p})$, Enhancer I/X promoter (EnI/Xp), Enhancer II/core promoter (EnII/Cp)) are vital for HBV synthesis, and the host factors are indispensably required for HBV expression and replication [4, 5]. Therefore, it is crucial to explore the host-virus interaction in hepatocellular carcinoma (HCC).

MicroRNAs (miRNAs), a type of RNA ( 22 nucleotides) with no translation ability, are demonstrated to negatively regulate the target gene expression [6]. MiRNAs have been demonstrated to be involved in kinds of physiological or pathological changes in human diseases [7]. Convincing evidence shows the association between host miRNAs and virus infection [8]. Many miRNAs, such as miR-185-5p [9] and miR-101 [10], were reported to suppress the amplification of $\mathrm{HBV}$ in vitro. Tu et al. illustrated that miR-212-3p overexpression decreased cell growth and enhanced apoptosis in HCC via inhibiting FOXA1 expression [11]. However, the underlying molecular mechanism of miR-212-3p in regulating HBV replication is still undefined.

Nuclear factor I (NFI) was initially reported as a cellular factor for adenovirus replication [12]. NFI contains four genes (NFIA, NFIB, NFIC, NFIX) to form 
homodimers or heterodimers which have affinity to stabilize and specify DNA [13]. Accumulating evidence discloses that NFIA is related to HBV replication and gene transcription $[14,15]$. Nevertheless, the mechanism of NIFA to regulate HBV replication was rarely documented. In the present research, we attempted to investigate the interaction between host factors miR-212-3p, NFIA and HBV, providing a new direction for elucidating the mechanism of persistent infection of HBV in HCC.

\section{Materials and Methods}

\section{Cell culture}

Human hepatoma HepG2 and HepG2.2.15 cells (contains 2 head to tails stably integrated HBV of the D-genotype) (Procell, Wuhan, China) were cultured in Dulbecco's modified Eagle's medium (DMEM; Solarbio, Beijing, China) supplemented with $10 \%$ fetal bovine serum (FBS; Thermo Fisher Scientific) in 5\% $\mathrm{CO}_{2}$ incubator at $37^{\circ} \mathrm{C}$.

\section{Plasmid construction and transfection}

The cDNA sequence of NFIA was amplified and subcloned into the downstream of pcDNA3.1 vector (Invitrogen) to construct NFIA-overexpressing plasmid (pcDNA-NFIA), and pcDNA3.1 empty vector (pcDNA) was utilized as the negative control. Small interfering RNA (siRNA) antagonistic to NFIA (si-NFIA) and scrambled negative control (si-NC) were obtained from Hanbio (Shanghai, China). miR-212-3p mimics (miR-212-3p), miR-212-3p antagonist (anti-miR-212-3p), and respective scrambled oligomer sequences (miR-NC, anti-miR-NC) were synthesized 
by Sangon Biotech (Shanghai, China). The wild-type or mutant untranslated regions (3'UTR) fragment of human NFIA containing the predictive binding sites with miR-212-3p were amplified and then subcloned into the downstream region of pGL3 (Promega, Madison, WI, USA) to obtain the recombinant luciferase reporter, namely NFIA-WT and NFIA1-MUT. The four HBV promoter reporters were purchased from Sangon Biotech.

qRT-PCR assay

Total RNA extraction was performed using TRIzol reagent (Invitrogen). The cDNA was synthesized by random primers using Moloney murine leukemia virus reverse transcriptase (Promega). SYBR Green PCR Master Mix (Ambion, Carlsbad, CA, USA) was applied to complete the quantitative PCR. MiR-212-3p was normalized by U6 small nuclear RNA, while NFIA was standardized by $\beta$-actin. Also, the levels of HBV DNA copies were determined by qRT-PCR as Ma et al. previously reported [16]. The sequences of primers were presented in Table 1.

\section{HBV replication and Southern blot assay}

HBV core particle-associated DNA was detected by Southern blot as previously reported $[17,18]$.

\section{ELISA assay}

ELISA kits (TW-regent co., LTD, Shanghai, China) were applied to examine the productions of $\mathrm{HBsAg}$ and $\mathrm{HBeAg}$ in the culture supernatant in accordance with the manual.

\section{Dual luciferase reporter assay}


The luciferase reporter constructs NFIA-WT or NFIA-MUT were transfected into HepG2.2.15 cells together with miR-212-3p, anti-miR-212-3p or respective control. At $48 \mathrm{~h}$ after transfection, the luciferase activity was measured using Luciferase Reporter Assay System (Promega), with Renilla luciferase activity as a reference.

\section{RNA binding protein immunoprecipitation (RIP) assay}

The binding specificity between NFIA and miR-212-3p was confirmed using Magna RNA immunoprecipitation kit (Millipore, Billerica, MA, USA). Briefly, cell lysis was performed in RIP lysis buffer comprising protease and ribonuclease inhibitors. Subsequently, $100 \mu \mathrm{L}$ samples were cultured with magnetic beads coupled with anti-Ago2 (Millipore) or anti-IgG (Millipore). Then the samples were co-cultured with protease $\mathrm{K}$ to decompose the protein content and facilitate the isolation of immunoprecipitated RNA. qRT-PCR was used to monitor the levels of NFIA in the precipitates.

\section{Western blot assay}

Protein samples from HepG2.2.15 cells were extracted using RIPA buffer (Solarbio, Beijing, China), and then separated by SDS-PAGE. Subsequently, the protein bands were transferred onto a polyvinylidene fluoride (PVDF) membrane (Millipore). Following incubation in the blocking buffer $(0.1 \%$ Tween-20 Tris buffer solution with 5\% skim milk; TBST) for $1 \mathrm{~h}$, the membrane was cultivated with moderate concentrations of primary antibodies against NFIA or $\beta$-actin (Cell Signaling technology, Boston, Massachusetts, USA) at $4{ }^{\circ} \mathrm{C}$ overnight. Then the membrane was incubated with appropriate amount of secondary antibody for $2 \mathrm{~h}$ at $37^{\circ} \mathrm{C}$. Finally, the 
chemiluminescence intensity was determined using enhanced chemiluminescence luminator (Bio-Rad Laboratories, Shanghai, China). $\beta$-actin was considered as an internal reference.

\section{Cell viability detection}

The viability of HepG2.2.15 cells was measured by MTT analysis. Briefly, cells $\left(1 \times 10^{5}\right)$ were planted in 96-well plates and maintained at standard condition. At indicated time points, $10 \mu \mathrm{L}$ of MTT (Sigma-Aldrich, St. Louis, MO, USA, $12 \mathrm{mM}$ ) solution was added into the wells and cultured for $4 \mathrm{~h}$ at $37^{\circ} \mathrm{C}$. After aspirating the supernatant, $150 \mu \mathrm{L}$ of dimethyl sulfoxide (DMSO) was supplemented to dissolve the generated formazan. A Multiscan Spectrum (Bio-Rad Laboratories) was utilized to test the absorption value at $490 \mathrm{~nm}$.

\section{Flow cytometry for apoptotic rate analysis}

Annexin V-Alexa Fluor 647/PI apoptosis detection kit (Fcmacs, Jiangsu, China) was used to determine the apoptotic rate in accordance with the manufacturer's manual. Briefly, cells were digested with $0.05 \%$ trypsin (Sigma-Aldrich) at $37^{\circ} \mathrm{C}$ and centrifugated at $200 \times \mathrm{g}$ for $3 \mathrm{~min}$. After washing twice with $0.01 \mathrm{M}$ cooling PBS, the re-suspended cells were labelled with Annexin V-fluorescein isothiocyanate (FITC) and propidine iodide (PI) for $20 \mathrm{~min}$. Apoptosis analysis was carried out by using a FACSCalibur flow cytometer (BD Biosciences, San Diego, CA, USA).

\section{Statistical analysis}

All experimental results were presented as mean \pm standard deviation (SD) and analyzed using SPSS19.0 software. Significant difference was evaluated by Student 
$t$-test or one-way analysis of variance (ANOVA). Difference is regarded as significant when $P<0.05$.

\section{Results}

\section{Inhibition of $\mathrm{HBV}$ replication and expression by miR-212-3p}

Firstly, the expression difference of miR-212-3p in HepG2 and stable HBV-producing hepatocarcinoma cells (HepG2.2.15) was assessed by qRT-PCR. As shown in Fig. 1A, the level of endogenous miR-212-3p was reduced in HepG2.2.15 cells compared to that in HepG2 cells. The transfection efficiency was confirmed by qRT-PCR, indicated by the dramatical up-regulation of miR-212-3p level in HepG2.2.15 cells transfected with miR-212-3p mimics or apparent down-regulation of miR-212-3p level in HepG2.2.15 cells transfected with anti-miR-212-3p (Fig. 1B). As you can see from Fig. 1C, it was evident that the level of HBV DNA copies in the culture supernatant was decreased by miR-212-3p overexpression, while suppression of miR-212-3p had the opposite effect. As presented by southern blot assay, decreased HBV replicative intermediates were observed in HepG2.2.15 cells with miR-212-3p overexpression; meanwhile, HBV replicative intermediates were markedly augmented in anti-miR-212-3p group (Fig. 1D). To evaluate the changes of HBV expression mediated by miR-212-3p, the levels of HBsAg and HBeAg were detected in the supernatant. The ELISA results exhibited that the levels of HBsAg and $\mathrm{HBeAg}$ were both strikingly declined in miR-212-3p-treated group, while remarkably enhanced in by the down-regulation of miR-212-3p (Fig. 1E and 1F). Above all, miR-212-3p was implicated in the suppression of HBV replication and expression. 


\section{Verification of NFIA as a direct target of miR-212-3p}

To elucidate the mechanism of miR-212-3p in suppressing HBV replication, two algorithms, including TargetScan (http://www.targetscan.org/) and Pictar (http://pictar.mdc-berlin.de/), were used to search for the potential targets of miR-212-3p. Both the results from web-based softwares found NFIA, one important regulator of multiple viruses, was the candidate of miR-212-3p. Besides, putative target sites for miR-212-3p had not been detected by the computational programs within the HBV genome (Data unshown). The complementary binding sites between NFIA 3'UTR and miR-212-3p were displayed (Fig. 2A). Transfection of miR-212-3p resulted in a significant reduction of the luciferase activity of WT-NFIA reporter, while the luciferase activity of WT-NFIA reporter was notably elevated in by miR-212-3p inhibitor; however, the luciferase activity of MUT-NFIA reporter had no markedly fluctuation in any treatment group (Fig. 2B and 2C). In addition, the specific binding between miR-212-3p and NFIA was further determined depending on RIP analysis. As presented in Fig. 2D, miR-212-3p overexpression led to an obvious enrichment of NFIA mRNA in Ago2 immunoprecipitation complex compared with that in IgG control group. Moreover, the mRNA level of NFIA was apparently repressed in miR-212-3p mimics group but enhanced in miR-212-3p inhibitor group (Fig. 2E). Also, the Western blot assay displayed the similar results (Fig. 2F). All these results indicated that NFIA was a bona-fide target for miR-212-3p. 
In order to determine whether NFIA was associated with HBV replication and expression, NFIA overexpression or knockdown was performed in HepG2.2.15 cells by transfection with pcDNA, pcDNA-NFIA, si-NC or si-NFIA. qRT-PCR and western blot results manifested that both the mRNA and protein levels of NFIA were elevated in pcDNA-NFIA-transfected cells, while decreased in si-NFIA-transfected cells (Fig. 3A and 3B). Moreover, NFIA overexpression resulted in increased HBV DNA copies, while NFIA knockdown reduced HBV DNA copies (Fig. 3C). Furthermore, increased HBV replicative intermediates were observed following the transfection of pcDNA-NFIA, whereas the contrary effect was found in si-NFIA group (Fig. 3D). Also, transfection of pcDNA-NFIA resulted in a promotion of HBsAg and $\mathrm{HBeAg}$ levels in the supernatant, while the concentrations of $\mathrm{HBsAg}$ and $\mathrm{HBeAg}$ were strikingly decreased by depletion of NFIA (Fig. 3E and 3F). These data suggested that NFIA facilitated HBV replication and expression.

NFIA overexpression reversed the suppressive effect of miR-212-3p on HBV replication and expression

To further confirm whether miR-212-3p suppressed HBV replication and expression through mediating NFIA expression, miR-NC + pcDNA, miR-NC + pcDNA-NFIA, miR-NC + pcDNA-NFIA-MUT, miR-212-3p + pcDNA, miR-212-3p + pcDNA-NFIA, or miR-212-3p + pcDNA-NFIA-MUT were transfected into HepG2.2.15 cells. Results showed that pcDNA-NFIA-mediated increase of $\mathrm{HBV}$ progeny, HBV replicative intermediates, as well as HBsAg and HBeAg concentrations was abrogated due to the miR-212-3p transfection, while restoration of NFIA expression reversed the 
suppressive effect of miR-212-3p on HBV transcripts, HBV replication, and HBsAg and HBeAg levels (Fig. 4A-4D). Moreover, pcDNA-NFIA-MUT transfection displayed similar stimulative effects in regulating HBV replication and expression as pcDNA-NFIA transfection, however, the changes induced by pcDNA-NFIA-MUT transfection were not affected by miR-212-3p overexpression (Fig. 4A-4D). Therefore, miR-212-3p inhibited HBV replication and expression through targeting NFIA.

\section{miR-212-3p inhibited HBV Enhancer I activity via repressing NFIA}

From the above results, it was obvious that miR-212-3p repressed HBV replication and expression partly via suppressing NFIA. To illustrate whether miR-212-3p exerted inhibitory effects on HBV replication and expression by HBV enhancers or promoters, the correlation between miR-212-3p and HBV promoter activities was firstly investigated. As a result, transfection of miR-212-3p remarkably decreased the Enhancer I/X promoter (EnI/Xp)-regulated luciferase activities in comparison with miR-NC group, however, there was no significant change in the luciferase activities of other reporters between miR-212-3p and miR-NC groups (Fig. 5A). On the contrary, miR-212-3p inhibitor drastically improved EnI/Xp activities (Fig. 5B). Subsequently, the potential effects of NFIA on EnI/Xp activity were investigated in HepG2.2.15 cells. As indicated in Fig. 5C, overexpression of NFIA significantly elevated EnI/Xp activities compared with that in control group, while knockdown of NFIA repressed ENI/Xp activities. Furthermore, co-transfection of miR-212-3p + pcDNA or miR-212-3p + pcDNA-NFIA was performed to identify whether effects of miR-212-3p on the suppression of EnI/Xp activities were mediated by NFIA. NFIA 
restoration partially counteracted the suppressive effect of miR-212-3p on the luciferase activity of EnI/Xp (Fig. 5D). Consequently, the above results indicated that miR-212-3p suppressed HBV EnI/Xp activities by directly targeting NFIA.

miR-212-3p repressed cell growth via negatively regulating NFIA

Then, we further explore whether the effect of miR-212-3p on HBV pathogenesis was associated with cell proliferation. MTT assay disclosed that cell viability was greatly reduced by miR-212-3p overexpresson, while this effect was partly weakened with the introduction of pcDNA-NFIA (Fig. 6A). Flow cytometry assay presented that NFIA up-regulation mitigated miR-212-3p-induced apoptosis (Fig. 6B). Taken together, miR-212-3p impeded cell proliferation and promoted apoptosis by inhibiting NFIA expression.

\section{Discussion}

$\mathrm{HBV}$ infection is considered as a central reason for hepatocarcinogenesis. It is well known that host miRNAs are implicated in virus infection and replication $[7,19]$. In this project, we concentrate to understand the molecular mechanism of miR-212-3p in modulating HBV replication and HCC progression. Our data demonstrated that miR-212-3p inhibited HBV Enhancer I activity to regulate HBV expression, replication and HCC progression by targeting NFIA.

As reported, many miRNAs in host are involved in HBV infection or pathogenesis. For example, Fan et al. documented that HBV genome amplification in liver cancer cells was impeded by miR-185-5p [8]. Also, miR-101 [9], miR-370 [15], miR-539 [10] were identified to suppress HBV genome amplification. However, Wang et al. 
demonstrated that depletion of miR-146a decreased HBV replication and expression in HCC [20]. The different miRNAs caused the contrary results on HBV replication, which may implicate that miRNAs play different roles in the complex regulatory networks of HBV replication and transfection. In this study, overexpression of miR-212-3p restrained the levels of HBV DNA copies, core particle-associated DNA, HBsAg and HBeAg levels in HepG2.2.15 cells,, whereas miR-212-3p inhibitor exhibited the crosscurrent. The data illustrated that miR-212-3p blocked HBV expression, replication and tumor progression in HCC.

Nuclear factor I (NFI) was previously found to be associated with virus replication and viral genes transcription [12]. For example, Tian et al. indicated that NFIA modulated by miR-220c promoted HBV replication, demonstrated by demonstrated by the decrease of HBsAg and HBeAg, as well as the decline of DNA and RNA (pgRNA and total RNA) of HBV [14]. Fan et al. suggested that miR-370/NFIA axis regulate HBV gene replication and expression [15]. In this study, NFIA was verified as a direct target of miR-212-3p. Also, NFIA silencing inhibited the levels of HBV DNA copies, core particle-associated DNA, HBsAg and HBeAg in HepG2.2.15 cells. Meanwhile, NFIA overexpression presented the opposite tendency. Further restoration experiments demonstrated that NFIA overexpression regained the levels of HBV DNA copies, core particle-associated DNA, HBsAg and HBeAg in HepG2.2.15 cells blocked by miR-212-3p. To sum up, miR-212-3p inhibited HBV replication by targeting NFIA.

According to the previous documents, NFI play vital and complicated roles in 
regulating HBV replication through different HBV promoters/enhancers. For instance, NFIA promoted HBV Enhancer I activity, and was negatively regulated by miR-200c [14]. Guo et al. discovered that miR-372/373 negatively regulated NFIB expression to repress Enhancer I-Cp activities [21]. In our research, it was found that miR-212-3p strikingly suppressed EnI/Xp activity, but had no effect on S1p, S2p, EnII/Cp activity. Moreover, silencing of NFIA hindered Enhancer I activity. Consistently, NFIA was previously found to induce HBV transcription, gene expression and replication by enhancing HBV enhancer I activities [15]. Furthermore, restoration of NFIA expression recovered the Enhancer I activity inhibited by miR-212-3p. To conclude, miR-212-3p repressed HBV Enhancer I activity by targeting NFIA.

The tumor-suppressive effects of miR-212-3p have been demonstrated in several malignancies through different mechanisms, such as osteosarcoma [22, 23], glioblastoma multiforme [24], and colorectal cancer [25]. A recent research revealed that miR-212-3p decreased cell invasion in HCC through inhibiting connective tissue growth factor (CTGF) [26]. In our study, it was confirmed that NFIA overexpression counteracted the inhibitory effect on cell viability and the promotive effect on apoptotic rate in HepG2.2.15 cells caused by miR-212-3p mimics. The above results unraveled that miR-212-3p impeded cell growth by targeting NFIA in HCC.

In conclusion, miR-212-3p suppressed HBV gene expression, replication and tumor progression in HCC via decreasing HBV Enhancer I activity by targeting NFIA. Therefore, the new regulatory pathway may shed light on the mechanism of host-virus network. 


\section{Conflicts of interest}

The authors have no conflicts of interest to declare. 


\section{References}

1. Gish RG, Given BD, Lai CL, et al. Chronic hepatitis B: virology, natural history, current management and a glimpse at future opportunities. Antiviral Res 2015; 121:47-58.

2. You CR, Lee SW, Jang JW, Yoon SK. Update on hepatitis B virus infection. World J Gastroenterol 2014; 20:13293-13305.

3. Delius H, Gough N, Cameron C, Murray K. Structure of the hepatitis B virus genome. J Virol 1983; 47:337-343.

4. Dandri M, Locarnini S. New insight in the pathobiology of hepatitis B virus infection. Gut 2012; 6:i6-i17.

5. Seeger C, Mason WS. Molecular biology of hepatitis B virus infection. Virology 2015; 479:672-686.

6. Wahid F, Shehzad A, Khan T, Kim YY. MicroRNAs: synthesis, mechanism, function, and recent clinical trials. Biochim Biophys Acta Mol Cell Res 2010; 1803:1231-1243.

7. $\mathrm{Xu}$ YQ, Wang HL, Gao WK. MiRNA-610 acts as a tumour suppressor to depress the cisplatin resistance in hepatocellular carcinoma through targeted silencing of hepatoma-derived growth factor. Arch Med Sci 2019.

8. Guo YE, Steitz JA: Virus meets host microRNA. the destroyer, the booster, the hijacker. Mol Cell Biol 2014; 34:3780-3787.

9. Fan HX, Feng YJ, Zhao XP, He YZ, Tang H. MiR-185-5p suppresses HBV gene expression by targeting ELK1 in hepatoma carcinoma cells. Life Sci 
$2018 ; 213: 9-17$.

10. Wang Y, Tian H. miR-101 suppresses HBV replication and expression by targeting FOXO1 in hepatoma carcinoma cell lines. Biochem Biophys Res Commun 2017; 487:167-172.

11. Tu H, Wei G, Cai Q, et al. MicroRNA-212 inhibits hepatocellular carcinoma cell proliferation and induces apoptosis by targeting FOXA1. OncoTargets Ther 2015; 8:2227-2235.

12. Gronostajski RM. Roles of the NFI/CTF gene family in transcription and development. Gene 2000; 249:31-45.

13. Kruse U, Sippel AE. Transcription factor nuclear factor I proteins form stable homo - and heterodimers. FEBS Lett 1994; 348:46-50.

14. Tian H, He Z. miR-200c targets nuclear factor IA to suppress HBV replication and gene expression via repressing HBV Enhancer I activity. Biomed Pharmacother 2018; 99:774-780.

15. Fan H, Lv P, Lv J, et al. miR - 370 suppresses HBV gene expression and replication by targeting nuclear factor IA. J Med Virol 2017; 89:834-844.

16. Ma YX, Li D, Fu LJ, et al. The role of Moloney leukemia virus 10 in hepatitis B virus expression in hepatoma cells. Virus Res 2015; 197:85-91.

17. Chua PK, Tang FM, Huang JY, Suen CS, Shih C. Testing the balanced electrostatic interaction hypothesis of hepatitis B virus DNA synthesis by using an in vivo charge rebalance approach. J Virol 2010; 84:2340-2351.

18. Le Pogam S, Chua PK, Newman M, Shih C. Exposure of RNA templates and 
encapsidation of spliced viral RNA are influenced by the arginine-rich domain of human hepatitis B virus core antigen ( $\mathrm{HBcAg} 165-173)$. J Virol 2005; 79:1871-1887.

19. Lamontagne J, Steel LF, Bouchard MJ. Hepatitis B virus and microRNAs: Complex interactions affecting hepatitis $\mathrm{B}$ virus replication and hepatitis $\mathrm{B}$ virus-associated diseases. World J Gastroenterol 2015; 21:7375-7399.

20. Wang Y, Li Y. miR-146 promotes HBV replication and expression by targeting ZEB2. Biomed Pharmacother 2018; 99:576-582.

21. Guo H, Liu H, Mitchelson K, et al. MicroRNAs - 372/373 promote the expression of hepatitis $\mathrm{B}$ virus through the targeting of nuclear factor $\mathrm{I} / \mathrm{B}$. Hepatology 2011; 54:808-819.

22. Li H, Tian G, Tian F, Shao L. Long non-coding RNA TUG1 promotes osteosarcoma cell proliferation and invasion through inhibition of microRNA-212-3p expression. Exp Ther Med 2018; 16:779-787.

23. Xie C, Chen B, Wu B, Guo J, Cao Y. LncRNA TUG1 promotes cell proliferation and suppresses apoptosis in osteosarcoma by regulating miR-212-3p/FOXA1 axis. Biomed Pharmacother 2018; 97:1645-1653.

24. Liu H, Li C, Shen C,et al. MiR-212-3p inhibits glioblastoma cell proliferation by targeting SGK3. J Neurooncol 2015; 122:431-439.

25. Zhang J, Zhang Y, Li X, Wang H, Li Q, Liao X. MicroRNA-212 inhibits colorectal cancer cell viability and invasion by directly targeting PIK3R3. Mol Med Rep 2017; 16:7864-7872. 
26. Chen JQ, Ou YL, Huang ZP, et al. MicroRNA-212-3p inhibits the Proliferation and Invasion of Human Hepatocellular Carcinoma Cells by Suppressing CTGF expression. Sci Rep 2019; 9:9820. 


\section{Figure Legends}

Fig. 1 miR-212-3p suppressed HBV replication and expression. (A) The level of miR-212-3p in HepG2 or HepG2.2.15 cells was measured by qRT-PCR. The HepG2.2.15 cells were transfected with miR-NC, miR-212-3p mimics, anti-miR-NC or anti-miR-212-3p, then the levels of miR-212-3p (B) and HBV DNA copies (C) were monitored via qRT-PCR. (D) The core particle-associated HBV DNA in transfected cells was detected by Southern blot assay. The positions of relaxed circular (RC) and single-stranded (SS) DNAs were indicated. The levels of HBsAg (E) and $\mathrm{HBeAg}(\mathrm{F})$ in the supernatant of transfected cells were assessed via ELISA. ${ }^{*} P<0.05$, $* * P<0.01$.

Fig. 2 miR-212-3p negatively regulated NFIA expression. (A) The complementary sequences between NFIA 3'UTR-WT and miR-212-3p, as well as the mutant sequences of NFIA. (B-C) The luciferase activity of WT-NFIA or MUT-NFIA reporter in HepG2.2.15 cells transfected with miR-212-3p, miR-NC, anti-miR-212-3p, or anti-miR-NC was assessed via dual luciferase reporter assay. (D) The enrichment degree of NFIA in IgG or Ago2 immunoprecipitation complex of miR-212-3p- or miR-NC-transfected HepG2.2.15 cells was evaluated by RIP assay. (E-F) The effects of miR-212-3p overexpression or knockdown on NFIA mRNA and proteins were measured by qRT-PCR and western blot. $* * P<0.01$.

Fig. 3 NFIA promoted HBV replication and expression. The HepG2.2.15 cells were transfected with pcDNA, pcDNA-NFIA, si-NC or si-NFIA. (A and B) The mRNA and protein levels of NFIA in transfected cells were detected by qRT-PCR and 
western blot. (C) The level of HBV DNA copies in transfected cell was measured by qRT-PCR. (D) The level of core particle-associated HBV DNA in transfected cells was detected by Southern blot assay. The positions of relaxed circular (RC) and single-stranded (SS) DNAs were indicated. (E-F) The levels of HBsAg (E) and $\operatorname{HBeAg}(\mathrm{F})$ in the supernatant of transfected cells were evaluated via ELISA. $* P<0.05$, $* * P<0.01$

Fig. 4 miR-212-3p suppressed HBV replication and expression by targeting NFIA. The HepG2.2.15 cells were transfected with miR-NC + pcDNA, miR-NC + pcDNA-NFIA, miR-NC + pcDNA-NFIA-MUT, miR-212-3p + pcDNA, miR-212-3p + pcDNA-NFIA, or miR-212-3p + pcDNA-NFIA-MUT. (A) The level of HBV DNA copies in transfected cell was measured by qRT-PCR. (B) The level of core particle-associated HBV DNA in transfected cells was detected by Southern blot assay. The positions of relaxed circular (RC) and single-stranded (SS) DNAs were indicated. (C and D) The levels of HBsAgand HBeAg in the supernatant of transfected cells were evaluated via ELISA. $* P<0.05, * * P<0.01$.

Fig. 5 miR-212-3p inhibited HBV Enhancer I activity by targeting NFIA. (A and B) The luciferase activity of preS1 promoter (S1p), preS2 promoter (S2p), Enhancer $\mathrm{II} /$ core promoter $(\mathrm{EnII} / \mathrm{Cp})$ or Enhancer $\mathrm{I} / \mathrm{X}$ promoter $(\mathrm{EnI} / \mathrm{Xp})$ reporter in HepG2.2.15 cells transfected with miR-NC, miR-212-3p, anti-miR-NC or anti-miR-212-3p were examined via dual luciferase reporter assay. (C) The effect of NFIA overexpression or knockdown on the Enhancer I activity in HepG2.2.15 cells was evaluated by dual luciferase reporter assay. (D) The Enhancer I activity in 
HepG2.2.15 cells transfected with miR-NC, miR-212-3p, miR-212-3p + pcDNA or miR-212-3p + pcDNA-NFIA was tested via dual luciferase reporter assay. $* P<0.05$, $* * P<0.01$.

Fig. 6 miR-212-3p repressed cell proliferation and induced apoptosis by targeting

NFIA. The HepG2.2.15 cells were transfected with miR-NC, miR-212-3p, miR-212-3p + pcDNA or miR-212-3p + pcDNA-NFIA. (A) The cell viability of transfected cells was assessed by MTT assay. (B) The apoptotic rate of transfected cells was monitored through flow cytometry. $* P<0.05, * * P<0.01$. 
Table 1. Oligonucleotide sequences used in the present study.

\begin{tabular}{ll}
\hline Gene & Sequences \\
\hline miR-212-3p-F & 5'-GGTAACAGTCTCCAGTCA-3' \\
miR-212-3p-R & 5'-GCAATTGCACTGGATACG-3' \\
NFIA-F & 5'-AGGTCTTTACCCAGCACATCCTC-3' \\
NFIA-R & 5'-TCCACTTGACTGACTGCCACTTC-3' \\
pMov10-F & 5'-ACATTCTACATTGCCCGCTTCTTG-3' \\
pMov10-R & 5'-CTCCTTCCTCTATCCGATTGGTCAC-3' \\
GAPDH-F & 5'-TGTTCGTCATGGGTGTGAAC-3' \\
U6-R & 5'-ATGGCATGGACTGTGGTCAT-3' \\
GAPDH-R & 5'-CTCGCTTCGGCAGCACA-3' \\
\hline &
\end{tabular}

Abbreviation: NFIA, nuclear factor I A; F, forward; R, reverse. 
A

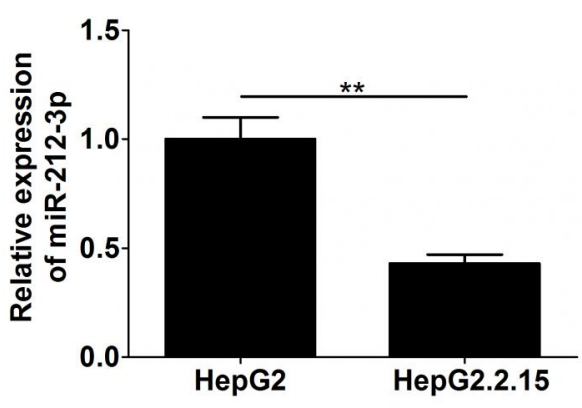

C

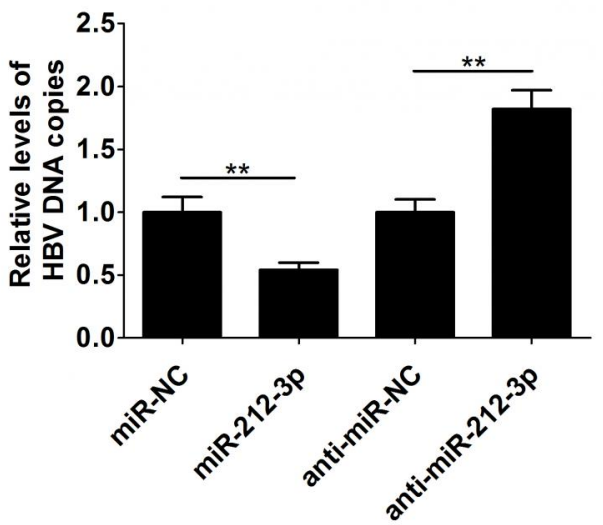

E

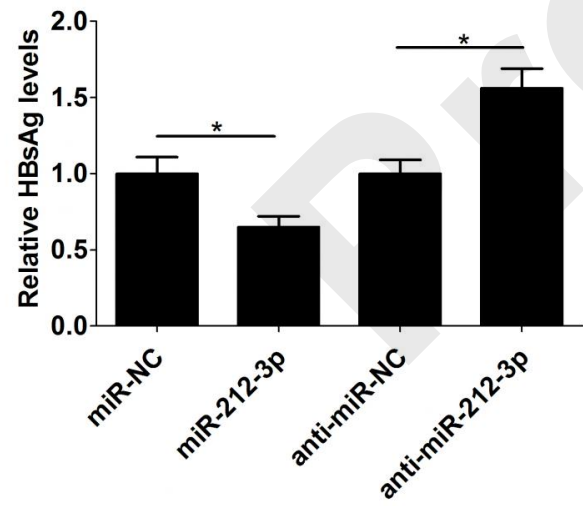

B

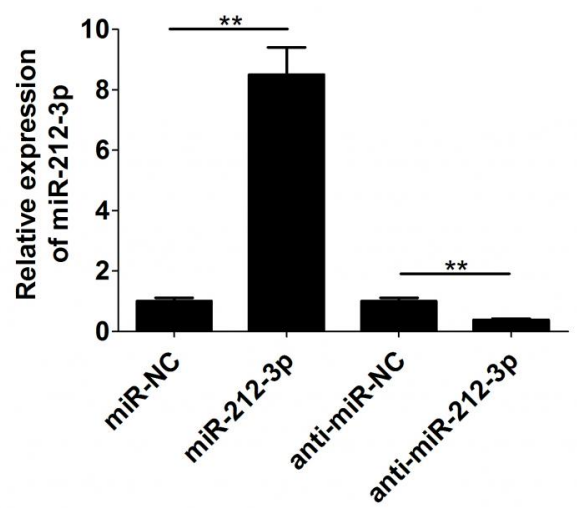

D

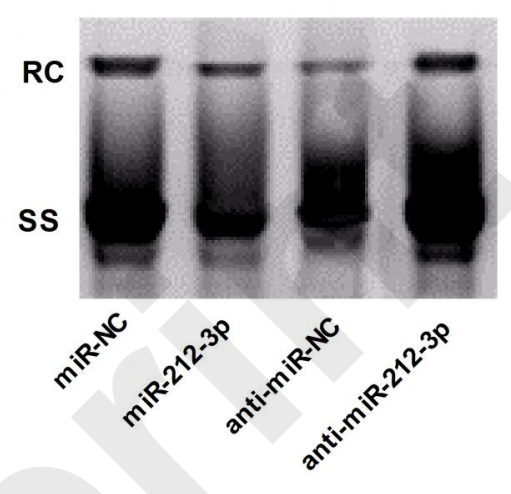

F

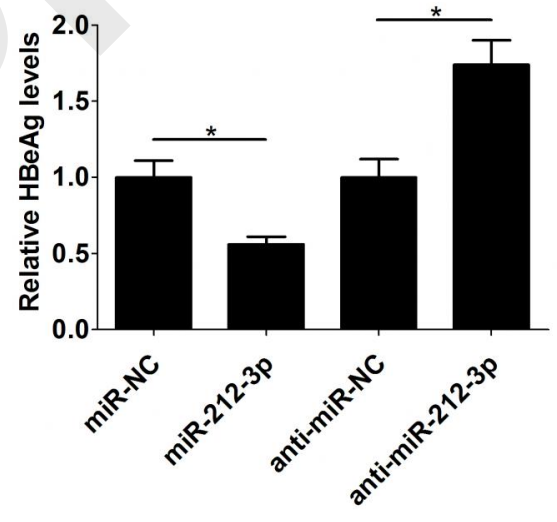


A

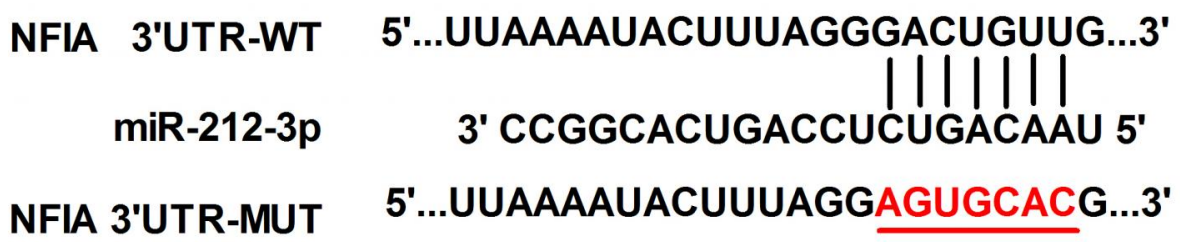

B

C
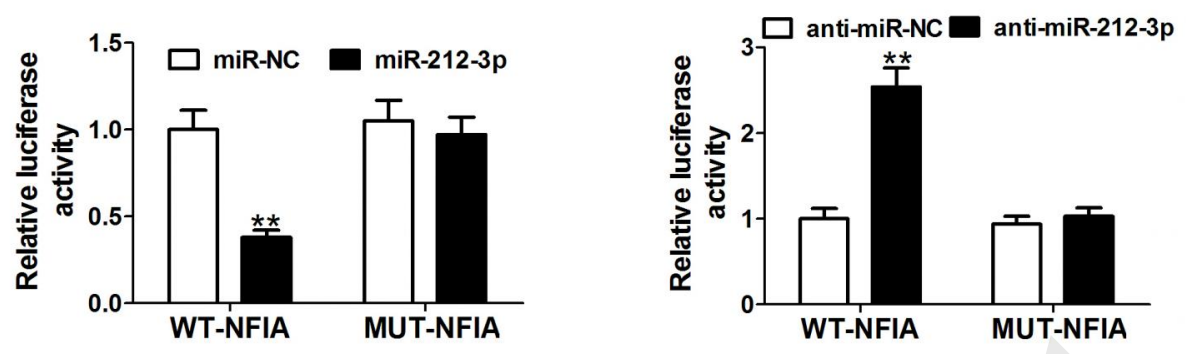

D

E
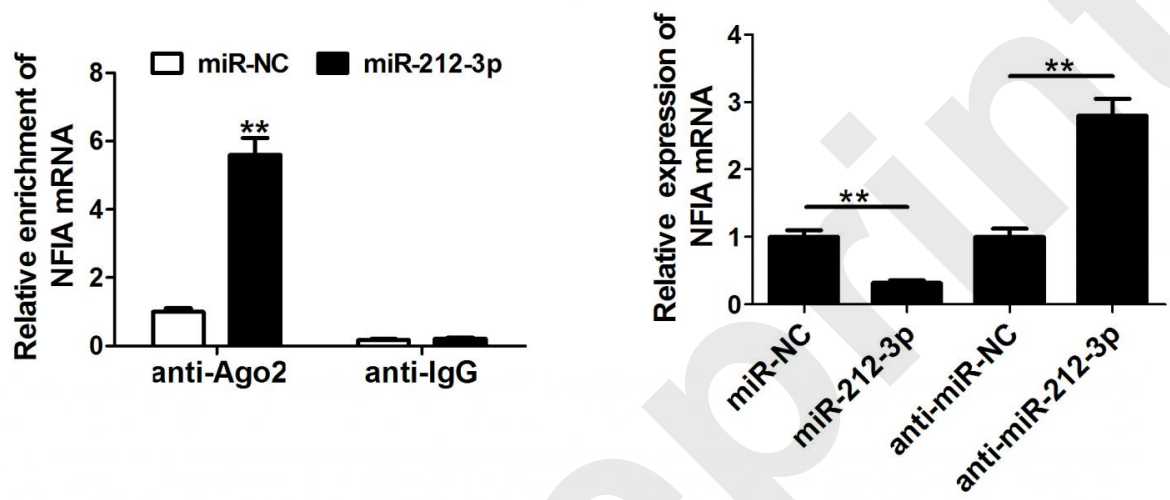

F

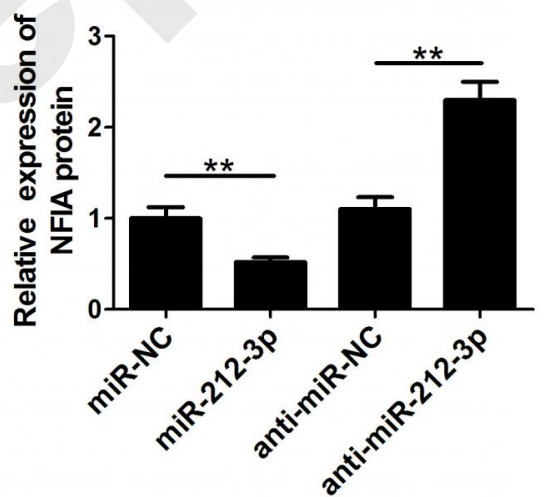


A

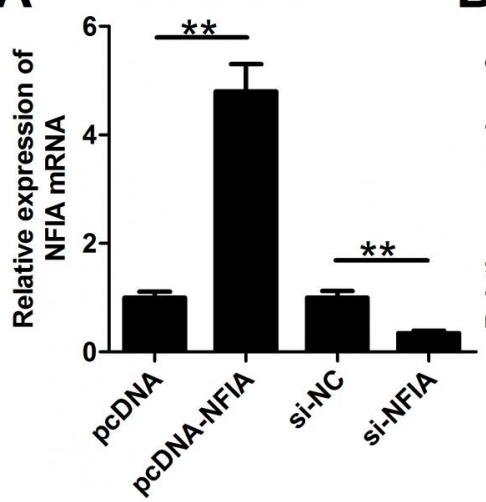

B

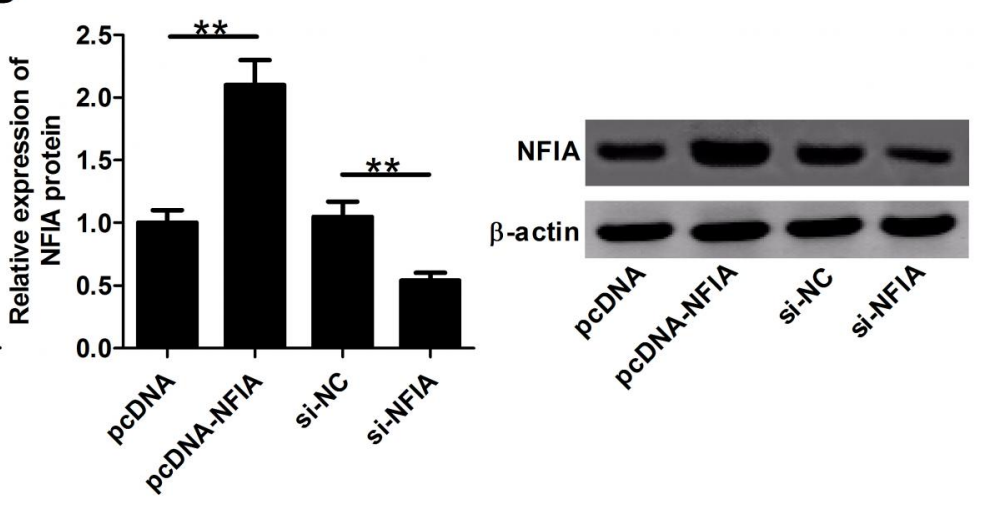

C

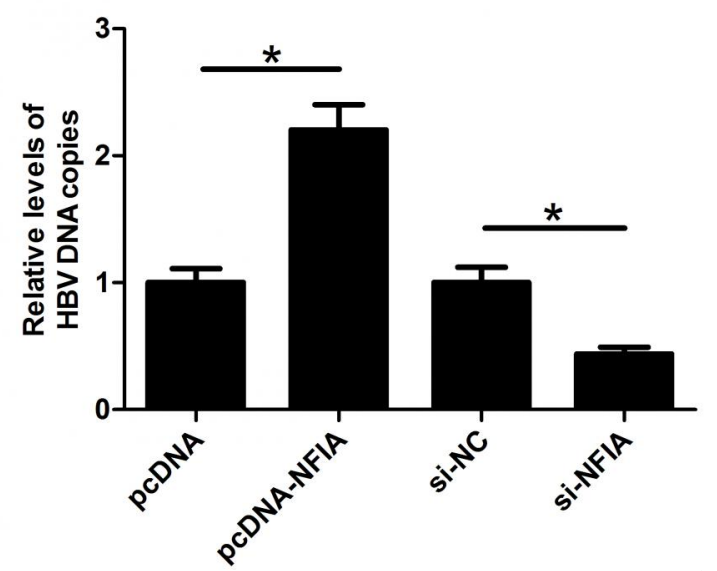

E

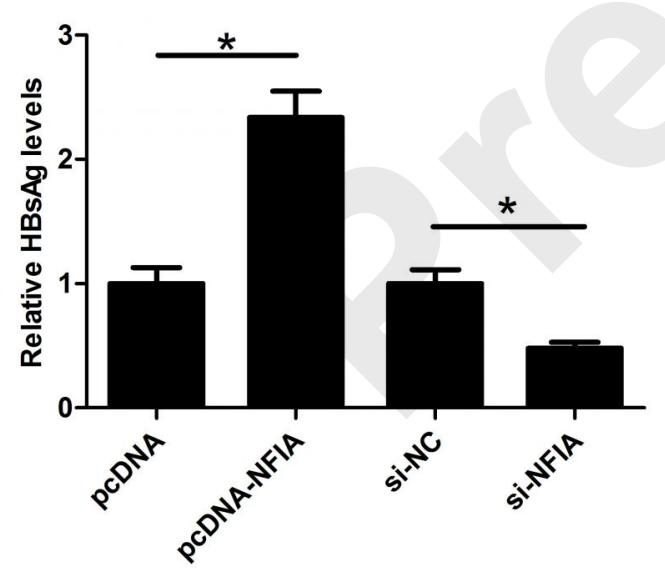

D

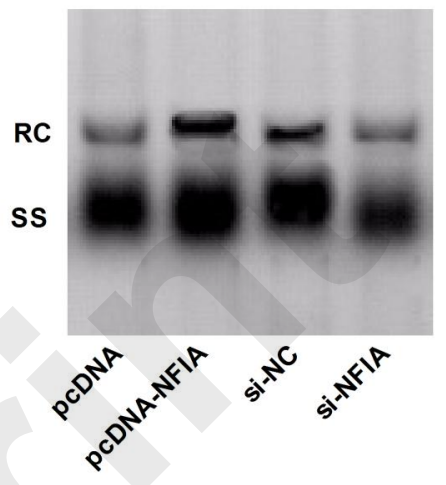

F

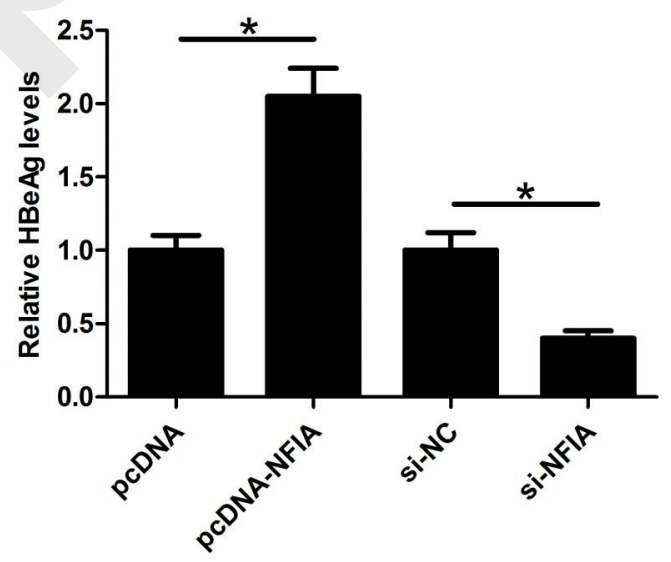


A
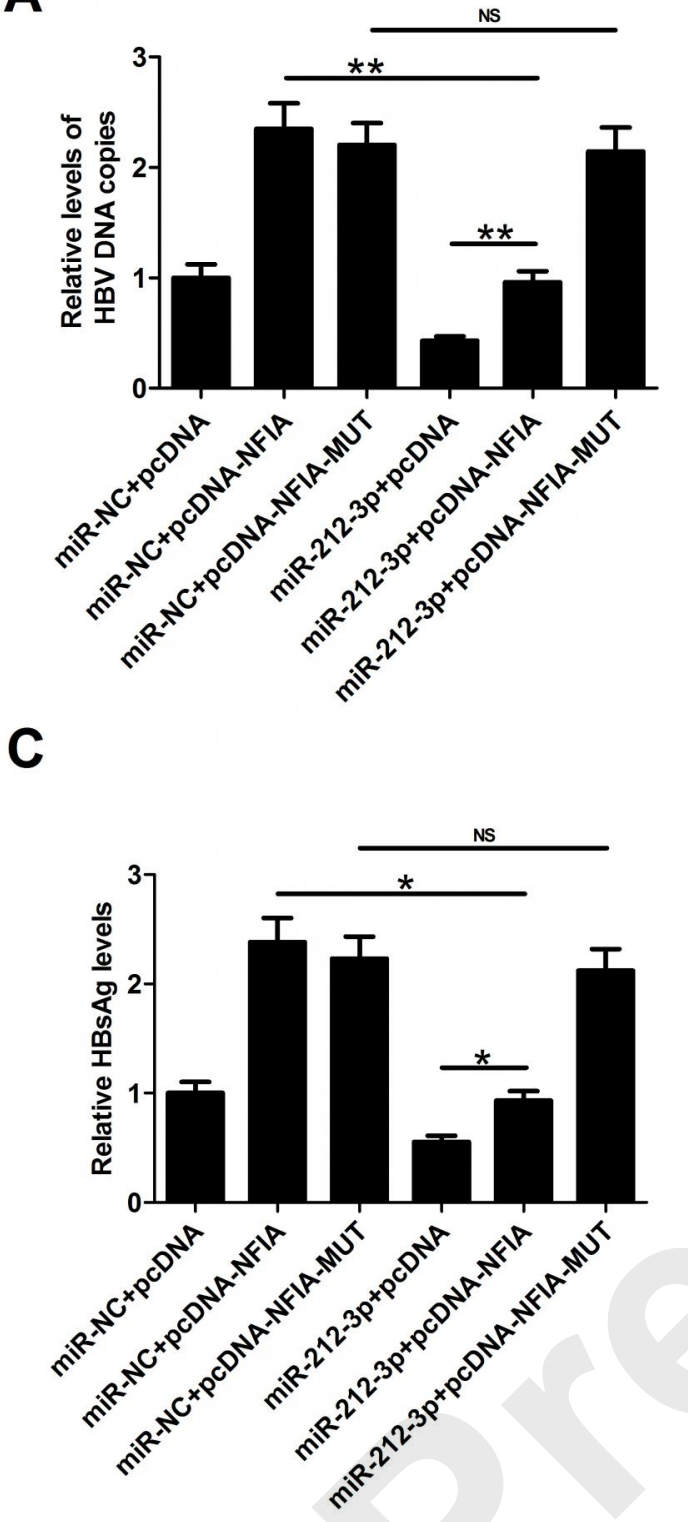

B

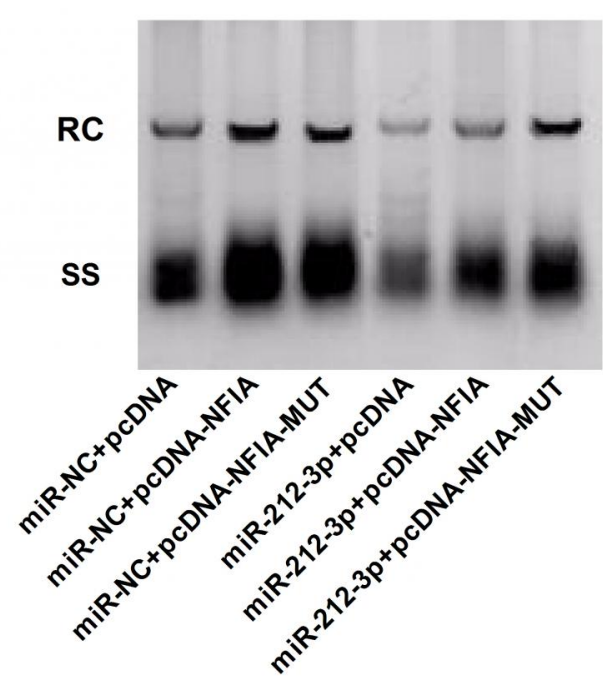

D

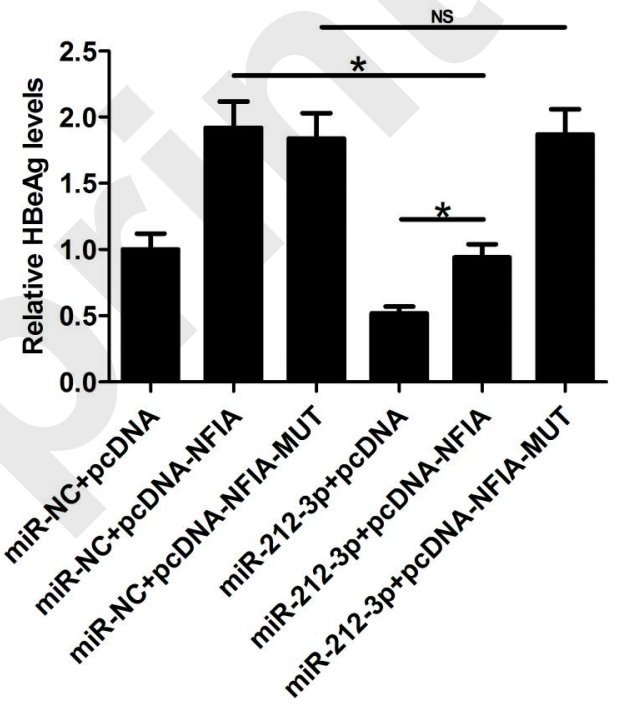


A

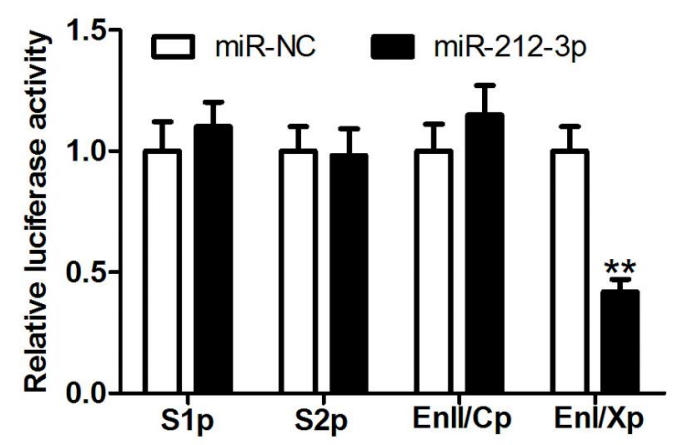

C

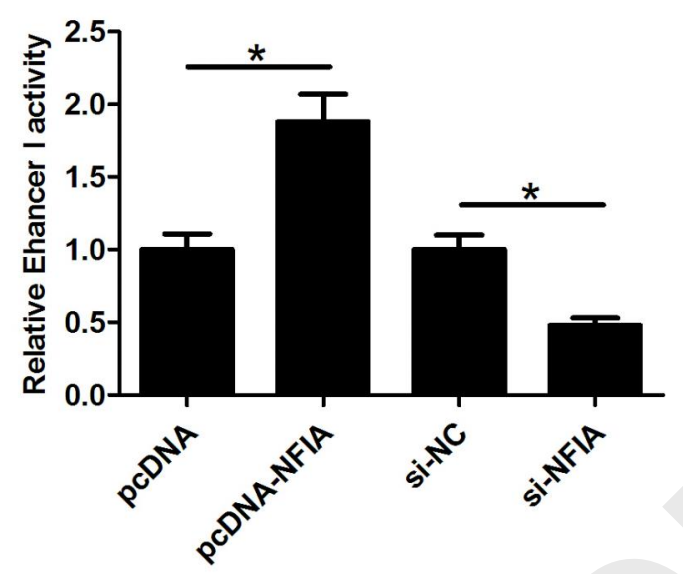

B

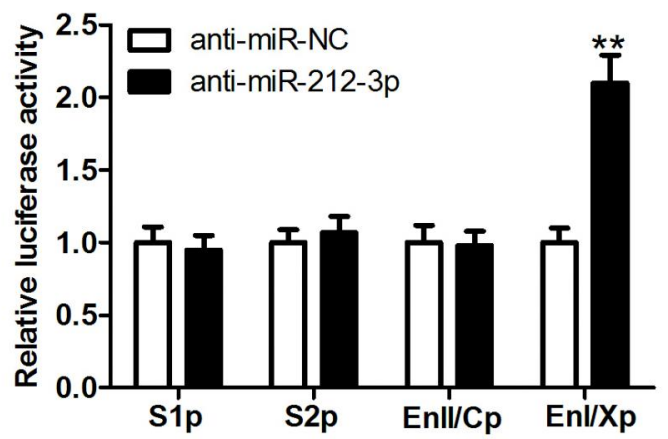

D

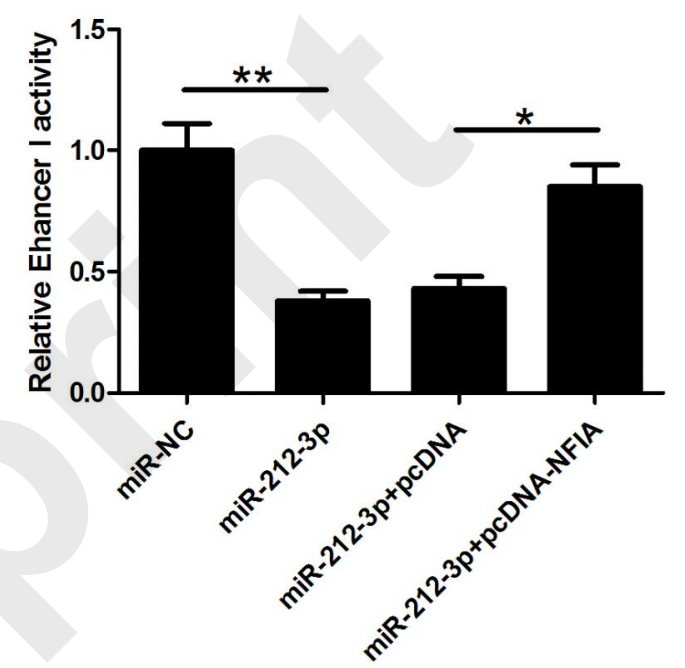



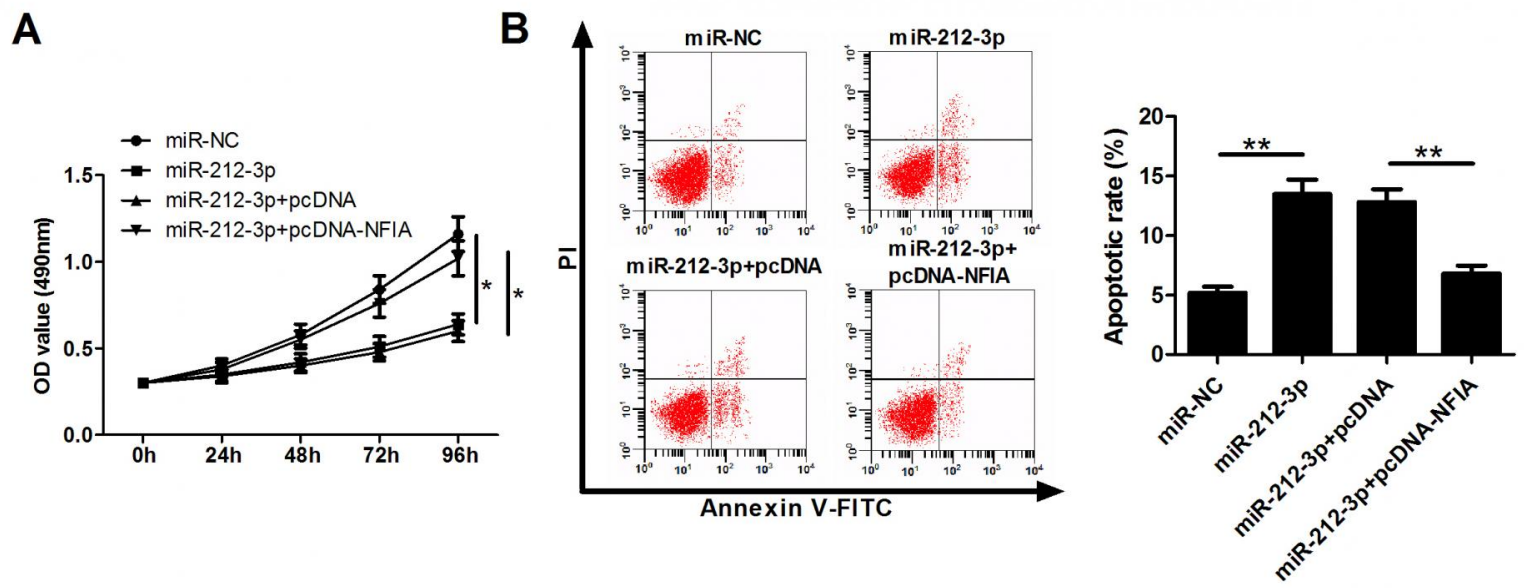